\title{
La preparación de los servicios de interpretación de lengua de signos: Contexto discursivo y esfuerzo físico
}

\author{
Preparing sign language interpretation services: Discourse context and \\ physical effort
}

Caridad Rosario Suárez-Martín ${ }^{1}$ (1) https://orcid.org/0000-0003-2770-5679

Universidad de Las Palmas de Gran Canaria

DELLCOS $\cdot$ C/ Pérez del Toro, $1 \cdot$ E35004 · Las Palmas de Gran Canaria (Spain)

David Rodríguez-Ruíz • (D) https://orcid.org/0000-0003-3617-3942

Universidad de Las Palmas de Gran Canaria

Departamento de Educación Física - Campus Universitario de Tafira $s / n \cdot$ E35004 · Las Palmas de Gran Canaria (Spain)

\section{RESUMEN}

La interpretación en lengua de signos demanda una gran competencia cognitiva y física en el intérprete de lengua de signos (ILS). Nuestro interés es estudiar, a través de la metodología de recogida de datos del análisis crítico del discurso, cómo preparan los ILSs sus contextos discursivos, incluyendo el esfuerzo físico existente. Realizamos un estudio mixto: cualitativo, del contexto discursivo y cuantitativo, evaluando la musculatura implicada, obteniendo como resultado que es de vital importancia que el ILS tenga la información con antelación para poder adaptarse rápidamente a diferentes ritmos del discurso y la tensión que las situaciones generan. Ya que deben enfrentarse a una amplia variedad de desafíos relacionados con las tareas, cognitivos y físicos, favoreciendo el posible riesgo de lesión muscular, es necesario para el ILS realizar actividades físicas compensatorias y preventivas. El conocimiento previo sobre el contenido a interpretar, los usuarios, características del lugar y otros aspectos a tener en cuenta, según el servicio, proporciona mejores herramientas para la realización del trabajo.

Palabras clave: lengua de signos, interpretación, metodología etnográfica, análisis crítico del discurso

\section{ABstract}

Sign language interpretation demands high cognitive and physical skills from the professional interpreter (SLI). The aim of this paper is to study how SLIs prepare their discoursive contexts, including physical effort, by collecting data from critical discourse analysis. With this purpose in mind, we have followed a twofold method, namely, a qualitative study of the context and a quantitative evaluation of the musculature involved. Our results indicate that it is vitally important for SLIs to have the relevant information in advance in order to be able to adapt quickly to different rhythms of speech and the tension that situation generates. Since they have to face the variety of cognitive and physical challenges of their tasks, with the concurrent risk of possible injury, SLIs must carry out compensatory and preventive physical activities. The information that the SLIs had in advance about the content to be intrerpreted, the users, the characteristics of the place and other relevant aspects, the nature of which depends on the specific service in question, is vitally important.

Keywords: sign language, interpretation, ethnographic methodology, critical discourse analysis 


\section{Introducción}

La interpretación de la lengua de signos abarca diferentes ámbitos en los que profesionales de este campo son necesarios para la comunicación entre la lengua propia de las personas que pertenecen a la comunidad sorda y la lengua oral del país en el que viven, como explican De Los Santos y Lara (2001), "El intérprete de lengua de signos podría definirse como aquel profesional competente en la(s) lengua(s) de signos y la(s) lengua(s) oral(es) de un entorno, capaz de interpretar los mensajes emitidos en una de esas lenguas a su equivalente de otra forma eficaz."

Los diferentes campos de trabajo de un intérprete de lengua de signos (ILS) hacen que éste deba enfrentarse a diversos contextos, siendo a veces conocidos y en otras ocasiones no, demandando altas competencias cognitivas y físicas, lo que influye en el proceso de interpretación (Dean y Pollard, 2011).

En este estudio, cobra mucha importancia, además del contenido a interpretar, el contexto en el que se produce. Para su análisis, factores como dónde tiene lugar la interpretación y si es conocido o no, si la interpretación es entre dos o más personas, la modalidad de discurso y con qué recursos cuenta para su preparación, son todos muy importantes y forma parte del contexto discursivo profesional en la interpretación en lengua de signos que los ILSs deben conocer para la consecución de su trabajo, así como de los usuarios que intervienen. Igualmente otro factor que influye, al contrario de las lenguas orales, y como indican Donner et al. (2016), "Sign language interpreting requires high levels of upper extremity postural deviation and repetitive motion", la postura física supone un gran esfuerzo físico en la actividad por parte del profesional. Por este motivo, además de las altas demandas cognitivas y psicosociales, tratándose de una lengua de un colectivo lingüístico en minoría, se da una alta demanda física y problemas de desorden musculoesquelécticos (MSD). Siguiendo a Fischer et al. (2012), "It is clear that the job of interpreting is associated with MSD pathology. Numerous studies have identified a high prevalence of pain, discomfort, and injury amongst this population”. Todo ello refleja que se requiere de una preparación física para afrontar las demandas de su trabajo.

Sin embargo, uno de los mayores problemas que reflejan los ILSs se refiere a la falta de preparación previa dada la contratación de los servicios con muy poco tiempo de antelación, citando a Nicodemus et al., (2014), "A fundamental principle held by professional American Sign Language-English interpreters is the critical importance of preparing for assignments; however, neither preparation strategies nor their efficacy have been studied in depth". El conocimiento a priori de la situación profesional a la que debe enfrentarse el ILS es de vital importancia para la consecución del trabajo. Significa, por un lado, realizar el análisis de la situación correspondiente del amplio contexto de interpretación y, por otro, la preparación física que el esfuerzo del proceso le va a exigir y que es inherente a una lengua viso-gestual como la lengua de signos.

Por tanto, son numerosos los factores, agentes e interacciones que se producen en un acto de interpretación, lo que denominamos en nuestro estudio el <<contexto discursivo>>, como dicen Winston y Roy (2015:95), "The context in discourse analysis can be separated somewhat arbitrarily into two types: internal context and external context." Además, debemos tener presente uno de los aspectos inherentes a la lengua viso-gestual con la que se trabaja, la alta demanda física, que requiere la mayor parte de las veces de una interpretación simultánea y en lugares que muchas veces no cumplen las condiciones adecuadas, en las que los usuarios de la lengua de signos y los intérpretes tienen que acomodarse a las circunstancias reales del momento para que la comunicación sea accesible. Consideramos que la metodología de recogida de datos cualitativa utilizada en el análisis crítico del discurso es la adecuada para poder abordar todo ese contexto discursivo desde distintas disciplinas (Morales López, 2011). 
Nuestro objetivo es estudiar cómo se preparan los ILS los contextos discursivos a los que se enfrentan. Como señala Van Dijk (1991:114), "El estudio del discurso es necesariamente una empresa interdisciplinaria". Para nosotros significa realizar una investigación mixta: por un lado, cómo prepara el ILS las situaciones discursivas a las que se enfrenta para la realización posterior del trabajo de interpretación en aquellos eventos reales que acontecen en la realización del trabajo, como propone Stubbs (1983:53), "Estudiar un acontecimiento de habla concreto en un marco social concreto." Por otro, conocer las características mecánicas del esfuerzo físico inherente al trabajo de interpretación de una lengua viso-gestual, mediante la tensiomiografía (TMG) de la principal musculatura implicada en la estabilización del hombro en el ILS.

Para ello, hemos realizado una investigación mixta: un estudio cualitativo, para la recogida de información del contexto discursivo a cuatro ILSs en dos ámbitos diferentes, y un estudio cuantitativo, donde se evaluó a catorce mujeres divididas en dos grupos: siete estudiantes del segundo curso del ciclo de interpretación en lengua de signos (GE) y siete profesionales en activo (GP). De la información recogida en la observación y en la entrevista, los aspectos más importantes en la preparación de los servicios fueron: análisis del lugar, preparación del contenido del acto, coordinación entre los ILSs para turnos con reparto del contenido y la coordinación con el equipo técnico. Del estudio cuantitativo, los resultados muestran que GP presentan un mayor nivel de rigidez muscular en todos los músculos evaluados $(\mathrm{p} \leq 0,05)$ que $\mathrm{GE}$, además de evidenciar una fatiga por sobresolicitación (exceso de uso del músculo) ante un movimiento repetido en el tiempo.

Nuestras conclusiones nos llevan a considerar que la información que se tenga con antelación sobre el texto, lugar de interpretación y los participantes, más el conocimiento de todo lo que acontece, proporciona mejores herramientas para la realización del trabajo de los ILSs. Además, los ILSs tienen que adaptarse rápidamente a una amplia variedad de desafíos en sus tareas, favoreciendo la disfunción asociada con trastornos de las extremidades superiores y posible riesgo de lesión si no se realizan actividades físicas compensatorias y preventivas que puedan evitar bajas laborales. Surge la necesidad de estudiar diferentes ámbitos discursivos y profundizar sobre las estrategias usadas por los ILSs, y la relación del esfuerzo físico con la preparación de los servicios de interpretación, a través de entrevistas y mediciones fisiológicas, más específicas, en distintos ámbitos de trabajo.

\section{Fundamentación Metodológica}

Se efectuó por un lado, un estudio cualitativo, recabando el mayor número de datos recogidos a través de metodología cualitativa del análisis crítico del discurso, acerca de la población estudiada sobre la preparación de sus servicios de interpretación. Por otro, un estudio cuantitativo para evaluar la musculatura de los profesionales y el esfuerzo físico que realizan.

\subsection{Estudio cualitativo}

\subsubsection{Diseño}

Para la recogida de información sobre la preparación de los servicios de interpretación se ha elegido como procedimiento la metodología de recogida de datos cualitativa del análisis crítico del discurso de las situaciones discursivas estudiadas. Como señala Alba-Juez (2009:27), "Thus, when it comes to data collection, our goals 
will guide us in the selection process and they are likely to lead us to choose different procedures", teniendo en cuenta nuestro objeto de investigación hemos elaborado una guía general del discurso para la elección del corpus, la recogida de la información y su análisis.

\subsubsection{Corpus seleccionado}

Se seleccionó deliberadamente una muestra que nos proporcionara información relevante acerca de lo que queríamos estudiar (Stubbs 1983:224). Esto es, una población representativa de la isla de Gran Canaria: cuatro ILSs de servicios generales (tres mujeres y un hombre) que corresponde al $20 \%$ de la población activa actual, formada por un lado, por una ILS con un año de experiencia profesional y por otro, tres ILSs con más de diez años de experiencia. Nuestro objetivo fue estudiar el proceso de preparación previo de servicios en los cuales se tiene la información y material acerca del mismo con tiempo de antelación suficiente y en los que se necesita la coordinación de diferentes agentes con el ILS.

Se realizó un convenio de colaboración con la Fundación Canaria para las Personas Sordas y sus Familias (FUNCASOR) para la observación de este tipo de profesionales en dos eventos: el primero corresponde al evento del Acto Institucional del Día de Canarias interpretado por tres ILSs y perteneciente a un ámbito específico: acto televisado sin presencia de personas signantes en la sala. El segundo corresponde a un servicio de una visita guiada a la Cueva Pintada de Galdar realizada por una ILS de servicios generales que, normalmente, se circunscriben a interpretaciones más individuales entre dos interlocutores y el ILS o pequeños grupos (De los Santos y Lara, 2001).

\subsubsection{Lugar de estudio}

Uno de los estudios se realizó en la sede de FUNCASOR en la isla de Gran Canaria. Esta fundación tiene como objetivo principal mejorar la calidad de vida de las personas con sordera y sus familias, sean o no signantes de la LS. Uno de los servicios para tal propósito es el servicio de ILSs denominado SILSE-FUNCASOR (http://funcasor.org/silse/). La observación del otro acto tuvo lugar en la sala sinfónica del Auditorio Alfredo Kraus de la ciudad de Las Palmas de Gran Canaria, durante los días 29 y 30 de mayo de 2016. Este evento, televisado por la Televisión Autonómica Canaria, requería de una gran coordinación entre los diferentes técnicos y responsables, incluyendo al equipo de interpretación en la propia escaleta de programación de la empresa pública organizadora, Canarias Cultura en Red, requiriendo de una gran colaboración entre los equipos de preparación y el grupo de ILSs.

\subsubsection{Instrumentos}

\subsubsection{Observación documental}

Una vez planteada la cuestión, hicimos una búsqueda en la literatura e investigaciones similares realizadas sobre el trabajo de interpretación de lenguas de signos, así como sobre el análisis crítico del discurso y, en concreto, en las lenguas de signos y en la interpretación a esa lengua. 


\subsubsection{Observación directa}

El objetivo fue hacer un estudio inicial, anotando y registrando lo acontecido, de forma descriptiva por un lado y por otro, interpretativa, siempre con el propósito final de extraer las pautas en la preparación de los servicios por parte de los ILSs. A modo de lo que Farclough, (2012:461) indica: "What data is selected, how it is collected, depend upon the project and object of research", fuimos creando nuestro formato de observación que contenía aquellos contextos y participantes que eran de nuestro interés.

\subsubsection{Entrevista libre de preguntas con respuestas abiertas}

Fue realizada a una ILS para obtener información sobre la preparación del servicio. Se realizó en su despacho, donde habitualmente prepara sus servicios con los recursos materiales y humanos adecuados como ordenador, acceso a Internet, glosarios y compañeras.

\subsubsection{Análisis de datos}

Para la recogida de toda la información, se tomó como formato de observación el siguiente cuadro de observación (Cuadro 1):

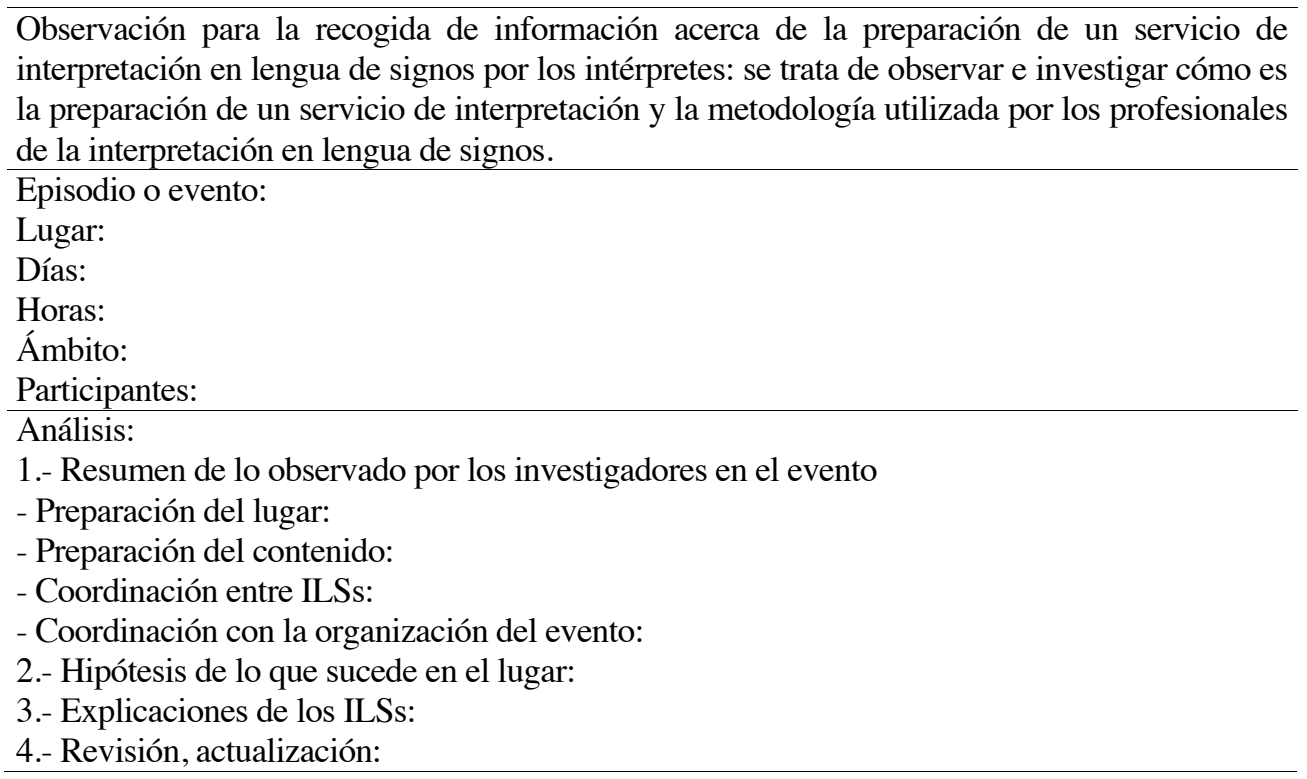

Cuadro 1. Modelo de guía de observación utilizado. Adaptado de Hernández et al. (2010).

Se llevó a cabo una Identificación de patrones de respuestas en la entrevista para la creación de instrumentos más precisos en la recogida de datos, como los cuestionarios, a partir de la categorización de las respuestas con más carga y frecuencia de mención y cuyos resultados fueron recogidos en el siguiente formato (Cuadro 2):

\begin{tabular}{l|l|l}
\hline Códigos & Patrones (respuestas con mayor frecuencia de mención) & Frecuencia de mención (n⿳0 de veces) \\
\hline & & \\
\hline
\end{tabular}

Cuadro 2. Cuadro de patrones de respuestas de la entrevista. Adaptado de Hernández et al. (2010).

Estos patrones fueron extraídos de la entrevista realizada a la ILS a partir de sus respuestas a las nueve preguntas planteadas (Cuadro 3): 
Preguntas planteadas en la entrevista a la ILSE sobre la preparación de su servicio: visita guiada a la Cueva Pintada de Gáldar

1. Tú me contaste que tenías un servicio de una visita guiada a la Cueva Pintada de Gáldar dentro de dos semanas. Cuéntame sobre el servicio.

2. ¿Qué vas a hacer ahora con esta documentación que tienes aquí?

3. A razón de lo que acabas de exponer, ¿por qué te preocupa menos?

4. ¿Conoces a los usuarios signantes?

5. Con el usuario que conoces, ¿tienes alguna relación o meramente de algún servicio o es alguien que conoces de tiempo?

6. Cuándo te enfrentas a ese texto por ejemplo, aparte de lo que comentabas antes, ¿qué más buscas dentro del texto?

7. Entonces ya tú conoces el contexto porque has ido antes. Has ido antes y has sido usuaria del servicio de guía.

8. El lugar no te preocupa entonces.

9. ¿Has consultado con otros ILS que hayan hecho este tipo de servicios?

Cuadro 3. Cuadro con el contenido de las preguntas de la entrevista a una intérprete.

\subsubsection{Aspectos éticos}

Se realizó un convenio de colaboración con FUNCASOR para el acceso a la entrevista de la ILS y la observación en la preparación antes del acto institucional.

Por otra parte y, previo a cada servicio, se contó con los permisos de cada ILS, con el compromiso por parte de los investigadores de confidencialidad y respeto de la información recogida, así como de su participación en el estudio.

\subsection{Estudio cuantitativo}

\subsubsection{Población}

Se evaluó a 14 mujeres, intérpretes de lengua de signos (ILS) con una edad media de 33,43 años $( \pm 5,88)$, una altura de $165,21 \mathrm{~cm}( \pm 5,50)$ y $68,00 \mathrm{Kg} .( \pm 22,93)$. Divididas en dos grupos, de las que 7 son estudiantes (Edad: 29,14 \pm 5,90 años; Altura: 164,14 \pm 5,52 cm; Peso: 68,57 \pm 15,31 Kg. y 8 meses de práctica) de segundo ciclo de interpretación en lengua de signos (GE) y 7 son profesionales (GP) en activo (Edad: 37,71 $\pm 5,88$ años; Altura: $166,29 \pm 5,50 \mathrm{~cm}$; Peso: 67,43 $\pm 22,93 \mathrm{Kg}$. y Años de práctica: 5,57 \pm 3,31) que trabajan en los ámbitos de educación y de servicios generales a la comunidad sorda de la isla de Gran Canaria. Las medidas se tomaron en el Laboratorio de Análisis y Planificación del Entrenamiento Deportivo de la Facultad de Ciencias Físicas y del Deporte de la Universidad de Las Palmas de Gran Canaria.

\subsubsection{Instrumentos}

Se utilizó la Tensiomiografía (TMG) para la evaluación de las características mecánicas (deformación radial máxima del vientre muscular - Dm, tiempo de activación - Td, tiempo de contracción - Tc, tiempo de relajación - Tr, tiempo de mantenimiento de la contracción - Ts). La medición se realizó con el sujeto tumbado en la camilla en posición prono para el dorsal ancho y en una silla especialmente diseñada con una separación del brazo de 30 grados frente al tronco para el deltoides medio y trapecio superior. En ambos casos el sujeto se encontraba totalmente relajado y sin realizar ningún tipo de esfuerzo, para ambos lados del cuerpo. La TMG 
mide la respuesta muscular mediante un sensor de desplazamiento (GK40, Panoptik, Ljubljana, Eslovenia) colocado sobre el vientre muscular del músculo seleccionado, orientado perpendicularmente a la dirección que tome el músculo en su deformación radial (Valencic y Knez, 1997). Para provocar la contracción del músculo se aplica una corriente eléctrica bipolar (100 mA), de 1 milisegundo de duración, a través de dos electrodos de superficie autoadhesivos bipolares (Compex Medical SA, Ecublens, Suiza) separados $4 \mathrm{~cm}$ de distancia desde el punto medio, sin afectar en ningún caso a los tendones de inserción (Knez y Valencic, 2000; Valencic, 2002; Simunic, 2003). La reproducibilidad del método y la validez del protocolo experimental que emplea la TMG han sido estudiadas en diferentes trabajos, mostrándose como una herramienta de alta precisión y efectividad para este tipo de estudio (Dahmane et al., 2000; Simunic, 2003; Belic et al., 2000; Simunic y Valencic, 2001; Krizaj et al., 2008; Tous-Fajardo et al., 2010; Rodríguez-Matoso et al., 2010; Carrasco et al., 2011; Simunic, 2012; Rey et al., 2012; Ditroilo et al., 2013; Simola et al., 2016).

\subsubsection{Musculatura analizada}

Se evaluó la musculatura superficial principalmente implicada en la estabilización del hombro: Trapecio Superior, Deltoides Medial y Pectoral Mayor. Los movimientos propios de interpretación del ILS requieren dicha estabilización, implicando directamente al Trapecio Superior (Hagberg et al., 1987; Lundberg et al., 2002; Delisle et al., 2005). Por otra parte, se ha decidido incorporar el Deltoides Medial y el Pectoral Mayor para poder comprobar su implicación en las acciones específicas del ILS durante la interpretación.

\subsubsection{Análisis estadístico}

Tras la realización de la prueba de normalidad (Shapiro-Wilk), se utilizó una ANOVA ONEWAY para muestras relacionadas en la comparativa entre grupos, con un ajuste Bonferroni a un nivel de significación de p $\leq 0,05$.

\section{Resultados}

\subsection{Estudio cualitativo}

El corpus seleccionado consta de cuatro ILSs de servicios generales, vinculados profesionalmente a FUNCASOR. Se analizaron dos procesos de interpretación, uno de ellos, un acto institucional celebrado en el Auditorio Alfredo Kraus de la ciudad de Las Palmas de Gran Canaria, con la asistencia de tres ILSs (dos mujeres y un hombre) con más de 10 años de experiencia laboral en la interpretación a LS. El otro proceso, corresponde a un servicio de interpretación en una visita guíada para un grupo de familias a la Cueva Pintada de Gáldar, realizada por una ILS de la misma fundación, mujer y con un año de experiencia profesional.

A continuación, pasamos a describir los resultados de la información recogida según la metodología diseñada:

3.1.1. De la observación directa en el Acto Institucional del Día de Canarias, destacamos los siguientes resultados de la información recabada acerca del proceso de preparación antes de los servicios de interpretación, a partir del modelo de guía de observación diseñado para ese evento (Cuadro 4): 


\title{
Observación para la recogida de información acerca de la preparación de un servicio de interpretación en lengua
} de signos por los ILS.

\author{
Episodio o evento: Celebración del Acto Institucional del Día de Canarias \\ Lugar: Auditorio Alfredo Kraus. Las Palmas de Gran Canaria \\ Días: 29 y 30 de mayo de 2016 \\ Horas: de 19:00 a 23:00 horas \\ Ámbito: Interpretación de conferencias: acto institucional. \\ Participantes: tres ILS con más de 10 años de experiencia \\ Análisis: \\ 1. Resumen de lo observado por la investigadora en el evento \\ - Preparación del lugar: preocupación por los aspectos técnicos al ser una grabación en directo \\ - Preparación del contenido: letra de las canciones, vídeos, gran contenido de topónimos del continente africano \\ - Coordinación entre intérpretes: buena colaboración entre los profesionales para la repartición de contenidos \\ - Coordinación con la organización del evento: compleja pero efectiva. Contacto mediante Whatsapp y correo electrónico \\ 2. Hipótesis de lo que sucede en el lugar: mayor preocupación por los aspectos técnicos y el contenido variado con \\ canciones y vídeos, más la dificultad de muchos topónimos desconocidos. \\ 3. Explicaciones de los ILSES: menos preocupación de los usuarios signantes ya que son indirectos a través de la \\ Televisión Canaria y no están presentes en la sala del auditorio. \\ 4. Revisión, actualización: identificar si hay una metodología presente en la preparación de este servicio común a los \\ intérpretes participantes. El papel de la preparación física previa o posterior.
}

Cuadro 4. Ejemplo de Observación recogida en el modelo guía.

La información recabada de la preparación del servicio de interpretación en lengua de signos por los ILS estuvo relacionada con los diferentes aspectos a tener en cuenta: adecuación del lugar del servicio, contenido, aspectos técnicos y coordinación entre ILSs y con la organización del evento. De la preparación del lugar, se tiene en cuenta el chequeo del lugar por cada ILS, su colocación en el espacio para interpretar designado y la comprobación del plano de la cámara. De la preparación del contenido del acto se señala: la preparación de canciones, los topónimos de países africanos, la fase de entrega de premios, los vídeos a exponer y los discursos principales.

La coordinación entre los ILSs para los turnos y el reparto del contenido se realizó según el mayor conocimiento por experiencia en el trabajo. En concreto, para el Acto del Día de Canarias, la distribución de las canciones y topónimos fue asumida por una de las compañeras la cual anteriormente había elaborado un glosario de topónimos de países y ciudades del mundo en FUNCASOR; el control de los aspectos técnicos por parte de otra de las ILSs del grupo, con experiencia en este tipo de acto por su trabajo en años anteriores con la empresa pública encargada de los aspectos técnicos; la organización con la Televisión Autonómica Canaria gracias al tercer ILS para el resumen de noticias accesibles a lengua de signos española y que conoce este medio de comunicación al ser el intérprete de esta cadena. Destacamos la comunicación a través de un grupo WhatsApp creado para el evento que facilitó la coordinación entre el grupo. La coordinación del equipo con la organización del evento se realizó a través de un grupo de WhatsApp creado, dos semanas antes de la celebración del evento, entre los ILSs y los responsables técnicos del acto, para la documentación y control de los aspectos técnicos necesarios, como la iluminación, el sonido, la televisión y la presentación del acto.

En relación al esfuerzo físico, de los cuatro ILSs, sólo uno hace calentamiento y estiramiento justo antes del comienzo del acto, pero en general, no se hace mención de la preparación física, ni previa ni posterior. 


\subsubsection{De la entrevista a una intérprete}

Una vez hecha la transcripción de la entrevista, introdujimos las respuestas en un primer cuadro de patrones, usando diferentes marcas para apreciar y contabilizar mejor las repeticiones y menciones en las respuestas. La entrevista se fue adaptando según los temas de interés de la ILS, que narró de forma detallada la preparación del servicio y sus opiniones respecto al mismo; del conocimiento del lugar en este caso, la visita guíada a la Cueva Pintada de Gáldar; de la organización y coordinación con la entidad a visitar para la obtención del contenido; y del conocimiento de los participantes, tanto oyentes como usuarios de la lengua de signos. A modo de ejemplo, mostramos en el siguiente cuadro, una selección resumida de patrones de esta entrevista, eligiendo las respuestas reiterativas para su categorización (Cuadro 5):

\begin{tabular}{|c|c|c|}
\hline Respuestas & $\begin{array}{l}\text { Patrones (respuestas con mayor } \\
\text { frecuencia de mención) }\end{array}$ & $\begin{array}{l}\text { Frecuencia de } \\
\text { mención } \\
\left(n^{0} \text { de veces }\right)\end{array}$ \\
\hline $\begin{array}{l}\text { Sobre el Servicio: } \\
\text {-No te puedo contar mucho del servicio (se rie) } \\
\text { porque no me han dado ninguna -documentación- } \\
\text {-como me estaba un poquillo así inquieta y demás por } \\
\text { el servicio ya hice algo de "investigación" } \\
\text {-pero eso "investigación" mía propia porque no tenía } \\
\text {-documentación- para prepararme } \\
\text { - mi compañera se puso en contacto con ellos para } \\
\text { solicitar esta -documentación- } \\
\text { - un resumen pequeñito y aquí lo tengo impreso para } \\
\text { preparármelo para el fin de semana y la semana que } \\
\text { viene. } \\
\text { - es una guía larga, bueno la visita es una hora y } \\
\text { media, pues claro yo necesito -tener mucha } \\
\text { información- }\end{array}$ & $\begin{array}{l}\text { Documentación/Información del servicio } \\
\text { Investigación } \\
\text { Preparación del servicio }\end{array}$ & $\begin{array}{l}4 \\
2 \\
2\end{array}$ \\
\hline
\end{tabular}

Cuadro 5. Ejemplo de un cuadro de patrones de recogida de respuestas resumidas de la entrevista. Adaptado de Hernández et al. (2010).

En la identificación de patrones de respuestas, anotamos algunas relevantes para una posterior creación de instrumentos más precisos en la recogida de datos, como los cuestionarios, a partir de la categorización de las respuestas con más carga y cuyos resultados podemos ver en el siguiente cuadro. A través de las nueve preguntas abiertas durante 20 minutos, y de las respuestas más reiterativas y mencionadas por la ILS, se recogieron los siguientes patrones de respuesta y su frecuencia de mención (Cuadro 6):

\begin{tabular}{llc}
\hline Códigos & $\begin{array}{c}\text { Patrones } \\
\text { (respuestas con mayor frecuencia de mención) }\end{array}$ & $\begin{array}{c}\text { Frecuencia de mención } \\
\left(\mathbf{n}^{\mathbf{0}} \text { de veces) }\right.\end{array}$ \\
\hline 01 & Preparación del servicio & 21 \\
02 & Análisis de la documentación & 21 \\
03 & Análisis del lugar de interpretación & 10 \\
04 & Recursos utilizados para la preparación & 10 \\
05 & Preocupación por el servicio & 9 \\
06 & Conocimiento/relación de los participantes & 6 \\
\hline
\end{tabular}

Cuadro 6. Cuadro para la clasificación de las respuestas más reiterativas. 


\subsection{Estudio cuantitativo}

Los resultados encontrados tras el estudio de la musculatura analizada, manifiestan diferencias en la adaptación de las características mecánicas evaluadas con la tensiomiografía en función de los años de práctica de la muestra (Tabla 1):

\begin{tabular}{|c|c|c|c|c|c|c|}
\hline & & & Profesionales & & antes & \\
\hline \multirow{7}{*}{$\begin{array}{l}\mathrm{Dm} \\
(\mathrm{mm})\end{array}$} & \multirow{3}{*}{ Deltoides } & & Media & SD & Media & $\mathrm{SD}$ \\
\hline & & derecha & 3,06 & 1,97 & 4,14 & 2,21 \\
\hline & & izquierda & 4,25 & 2,56 & 4,91 & 1,57 \\
\hline & \multirow[t]{2}{*}{ Dorsal } & derecha & 4,33 & 1,14 & 5,44 & 1,93 \\
\hline & & izquierda & 3,71 & 2,21 & 6,56 & 1,90 \\
\hline & \multirow[t]{2}{*}{ Trapecio } & derecha & 2,69 & 2,33 & 4,01 & 1,53 \\
\hline & & izquierda & 2,69 & 1,04 & 4,32 & 1,26 \\
\hline \multirow[t]{6}{*}{$\mathrm{Td}(\mathrm{ms})$} & \multirow[t]{2}{*}{ Deltoides } & derecha & 23,68 & 5,25 & 20,08 & 3,93 \\
\hline & & izquierda & 21,24 & 4,27 & 18,53 & 2,54 \\
\hline & \multirow[t]{2}{*}{ Dorsal } & derecha & 27,16 & 2,78 & 31,20 & 5,92 \\
\hline & & izquierda & 26,29 & 9,48 & 29,03 & 7,05 \\
\hline & \multirow[t]{2}{*}{ Trapecio } & derecha & 26,90 & 8,35 & 23,18 & 3,76 \\
\hline & & izquierda & 28,83 & 7,80 & 23,64 & 2,62 \\
\hline \multirow[t]{6}{*}{$\mathrm{Tc}(\mathrm{ms})$} & \multirow[t]{2}{*}{ Deltoides } & derecha & 14,24 & 2,58 & 14,91 & 3,59 \\
\hline & & izquierda & 15,66 & 0,78 & 14,87 & 0,99 \\
\hline & \multirow[t]{2}{*}{ Dorsal } & derecha & 20,45 & 4,33 & 24,69 & 8,29 \\
\hline & & izquierda & 24,63 & 10,09 & 27,77 & 11,20 \\
\hline & \multirow[t]{2}{*}{ Trapecio } & derecha & 17,20 & 2,81 & 20,09 & 5,22 \\
\hline & & izquierda & 31,46 & 19,51 & 22,39 & 12,18 \\
\hline \multirow[t]{6}{*}{$\operatorname{Tr}(\mathrm{ms})$} & \multirow[t]{2}{*}{ Deltoides } & derecha & 48,76 & 48,31 & 133,08 & 93,08 \\
\hline & & izquierda & 39,69 & 38,62 & 103,03 & 119,43 \\
\hline & \multirow[t]{2}{*}{ Dorsal } & derecha & 241,98 & 303,13 & 314,65 & 269,89 \\
\hline & & izquierda & 121,13 & 238,67 & 236,66 & 209,73 \\
\hline & \multirow[t]{2}{*}{ Trapecio } & derecha & 42,96 & 61,97 & 164,58 & 228,99 \\
\hline & & izquierda & 78,99 & 80,08 & 129,86 & 113,55 \\
\hline \multirow[t]{6}{*}{ Ts (ms) } & \multirow[t]{2}{*}{ Deltoides } & derecha & 443,59 & 247,03 & 355,07 & 327,86 \\
\hline & & izquierda & 627,05 & 384,21 & 292,33 & 216,41 \\
\hline & \multirow[t]{2}{*}{ Dorsal } & derecha & 808,61 & 295,52 & 492,59 & 268,11 \\
\hline & & izquierda & 695,34 & 252,39 & 429,60 & 280,51 \\
\hline & \multirow[t]{2}{*}{ Trapecio } & derecha & 481,76 & 298,64 & 437,17 & 305,12 \\
\hline & & izquierda & 508,30 & 216,06 & 444,00 & 216,55 \\
\hline
\end{tabular}

Tabla 1. Medias y Desviación típica (SD) de los resultados de los cinco parámetros de la TMG (Dm: Deformación máxima; Td: Tiempo de activación; Tc: Tiempo de contracción; Tr: Tiempo de relajación y Ts: Tiempo de mantenimiento de la contracción) para la musculatura evaluada, con un valor de significación $p \leq 0,05$. 


\section{Discusión}

\subsection{Estudio cualitativo}

De este estudio se destaca que, en la preparación de los servicios de interpretación a lengua de signos (LS), la información que se tenga con antelación sobre el lugar de interpretación y participantes es de vital importancia para la consecución del trabajo de los ILSs. A crucial aspect of interpreting source language discourse, whether spoken or written, is comprehension of the message (Holz-Mänttäri 1984) como se cita en Nicodemus et al. (2014:30). Por esta razón resulta muy importante la comprensión del mensaje y el previo análisis de los contenidos siendo el aspecto que más preocupa a los profesionales.

La coordinación entre los ILSs para los turnos y reparto del contenido así como la coordinación con el equipo de la organización del evento son fundamentales para la realización de un buen trabajo. En el servicio estudiado (acto institucional televisado), cobró mucha importancia el espacio y los aspectos técnicos debido a las características del servicio, con poca o nula asistencia de personas usuarias de la LS en el auditorio. Destaca en este estudio el uso de las nuevas tecnologías gratuitas y de fácil acceso, como el chat a través de WhatsApp, que permitió y facilitó la preparación, con antelación, del servicio de interpretación del acto institucional entre los distintos profesionales.

Sin embargo, los ILSs hacen poca mención a la preparación física, ni previa ni posterior, en ninguno de los dos tipos de preparación del trabajo, lo que significa una ausencia importante en nuestros datos. Sin embargo, el conocimiento de algunos de los factores que afectan al cansancio y las posibles afecciones en los ILSs, puede ayudar a minimizar el riesgo de lesiones y de cansancio, adoptando prácticas de trabajo adecuadas, a través del análisis del contexto discursivo de la situación de interpretación para la preparación por adelantado, permitiendo que el ILS pueda controlar las demandas a las que se enfrenta, tanto físicas, cognitivas como psicosociales (Fischer et al., 2012) y de la misma manera, puedan identificar el impacto del esfuerzo físico en su trabajo de interpretación, siendo la dimensión física inherente a la postura de trabajo, en la que menos se incide en la formación de los futuros intérpretes y, en la preparación de los servicios por parte de los profesionales en activo.

\subsection{Estudio cuantitativo}

Los resultados del estudio de la musculatura de las ILS muestran una adaptación a la actividad propia de la interpretación y, además, evidencian una diferencia clara entre grupos (GE vs GP), siendo estadísticamente significativa en algunos de los músculos evaluados (Tabla 1). Respecto a la Dm, encontramos diferencias estadísticamente significativas entre GE y GP, donde GP muestra un mayor nivel de rigidez muscular en todos los músculos evaluados que GE. Este nivel de rigidez se puede deber a la especialización de las personas evaluadas con más años de práctica (GP), según las afirmaciones realizadas por Rodríguez-Matoso et al. (2012) en su trabajo sobre las consideraciones metodológicas de la interpretación de los datos de TMG.

Por otro lado, el único estudio que se centra en la misma musculatura que nuestro trabajo, es el de García-García et al. (2016) pero con una muestra de women kayakers (mujeres kayakistas) de diferente nivel competitivo y, en este caso, el Dm observado en las mujeres especializadas es superior por un mayor volumen de la musculatura evaluada. Por tanto, se necesitaría poder comparar con personas no entrenadas para ver si este aumento de rigidez es a causa de la actividad propiamente o una fatiga debido a un daño por sobresolicitación (exceso de uso del 
músculo). Esta duda surge al estudiar los efectos del daño muscular sobre la musculatura del bíceps en un estudio de Hunter et al. (2012), donde se observó un descenso brusco de Dm al producirse dicho daño muscular. Valores de Dm por debajo de $3 \mathrm{~mm}$ en personas no especializadas en actividades explosivas son propensas a la lesión, (Rodríguez-Ruiz et al. 2012), dato que podemos observar en el grupo de GE para el trapecio en derecha $(2,69 \pm 2,33 \mathrm{~mm})$ y en izquierda $(2,69 \pm 1,04 \mathrm{~mm}) \mathrm{y}$, que enuncia una posible disfunción muscular que puede traer consecuencias de cese de la actividad por lesión correspondiente a la mayor exposición mecánica de estos profesionales (Fuerstein et al, 1997, Donner et al, 2016). Qin et al. (2008) argumentan que "The pain and dysfunction associated with upper extremity disorders hafe led to significant loss of work time for many interpreters and forced some of them to abandon the profesion entirely". El ILS durante su trabajo debe adaptarse rápidamente a los diferentes ritmos del discurso y a la tensión que las diversas situaciones generan.

Llama poderosamente la atención que en los valores de Tc y Td no haya diferencias entre los dos grupos. Lo que sugiere una rápida adaptación de los parámetros neurales a la actividad por parte de las estudiantes (GE). Podemos entender que la implicación del ILS en la interpretación hace que dicha musculatura deba adaptarse rápidamente a una amplia variedad de desafíos relacionados con las tareas. Como indican Qin et al. (2008): "In addition to the physical demands of signing, interpreting places considerable cognitive demand on the interpreter in that he/she often works in a highly dynamic environment and must quickly adapt to a wide variety of taskrelated challenges". La combinación de la tensión mental inherente a la tarea de interpretación y la suma de factores situacionales y ambientales pueden poner al ILS en una situación muy estresante y, en este sentido, el riesgo de que los ILSs desarrollen una disfunción músculoesquelética también ha sido claramente documentada (Feuerstein y Fitgerald, 1992; Fischer et al., 2012; Donner et al., 2016), debido a la carga que la musculatura de la parte superior del cuerpo (upper body) soporta durante la interpretación (Shealy et al., 1991; Marras y Shoenmarklin, 1993). Por un lado, los desafíos propios de la tarea de interpretación y por otro, las diferentes situaciones de interpretación a las que deben enfrentarse hacen que algunos ILSs abandonen pronto la profesión o bien causen bajas laborales con frecuencia.

Por último, analizando los parámetros Tr y Ts que podemos considerar como valores que reflejan una fatiga por sobresolicitación ante un movimiento repetido en el tiempo (Rodríguez-Matoso et al., 2012; Rodríguez-Ruiz et al., 2012; García-Manso et al., 2011 y 2012), se puede observar que Tr muestra valores superiores en GE respecto a GP, siendo esta diferencia estadísticamente significativa en Deltoides, tanto en derecha $(\mathrm{p}=0,039)$ como en izquierda $(\mathrm{p}=0,049)$. Por otro lado, Ts presenta valores superiores en GP respecto a GE, siendo estadísticamente significativo en ambos Dorsales (derecho: $p=0,042$ e izquierda: $p=0,047)$ y en Deltoides izquierdo $(p=0,050) y$, con una tendencia en derecho ( $\mathrm{p}=0,085)$. Los estudios de García-Manso et al. (2011 y 2012) nos dicen que un aumento de estos parámetros se debe al enlentecimiento de los procesos de relajación del músculo después del proceso de contracción-descontracción repetido en el tiempo y con poca recuperación entre uno y otro proceso. Este comportamiento es el que encontramos en las acciones técnicas de los deportes cíclicos de larga duración (García-Manso et al., 2011) y en acciones de fuerza ante distintas intensidades y volúmenes de trabajo (GarcíaManso et al., 2012). En el caso del ILS, los datos de nuestro estudio los reflejan en su actividad interpretativa con gran demanda tanto cognitiva como psicosocial y con poco control de las situaciones de interpretación (Donner et al., 2016). Por tanto, este valor de Ts en GP refleja una adaptación de las ILS a la posición de brazos levantados sin apoyo mantenida durante su actividad (Deslile et al, 2005) y que, junto con los valores de $\operatorname{Tr}$, nos hacen ver el riesgo de lesión si no se realizan actividades compensatorias y preventivas. 


\section{Conclusiones y futuras investigaciones}

En la preparación de un acto de interpretación de lengua de signos el conocimiento del contexto discursivo, por parte del ILS, proporciona mejores herramientas para la posterior realización del trabajo. Para estos profesionales es necesaria la información previa del servicio sobre aspectos tales como el espacio donde se realiza el servicio, los aspectos técnicos a tener en cuenta según la situación, la coordinación entre los ILSs para los turnos y reparto del contenido así como, en el caso de nuestro estudio, la coordinación con el equipo de la organización del evento.

La dimensión física inherente a la postura de trabajo es en la que menos se incide en la preparación de los servicios por parte de los profesionales en activo. Sin embargo, los resultados de la musculatura evaluada en los sujetos de nuestra muestra señalan que los ILSs tienen que adaptarse rápidamente a una amplia variedad de desafíos relacionados con las tareas, tanto cognitivas como físicas, favoreciendo la disfunción asociadas con los trastornos de las extremidades superiores y el posible riesgo de lesión si no se realizan actividades físicas compensatorias y preventivas con ejercicios de estiramiento y fortalecimiento muscular que puedan evitar un gran número de bajas laborales.

De las especiales características de las diferentes situaciones, abordadas por los profesionales, y las diferentes propiedades de estos contextos transculturales, tratándose de una lengua con un código viso-gestual que utiliza las manos y parte superior del cuerpo para su expresión, sería necesario realizar más estudios sobre el esfuerzo físico que requiere una situación de interpretación determinada triangulando datos que nos permitan dar cuenta del mismo como parámetro en relación a la preparación de los servicios de interpretación a lengua de signos.

Dado la variedad de las situaciones de interpretación en diferentes ámbitos como, por ejemplo, la universidad y la educación secundaria obligatoria, el movimiento asociativo de personas sordas, los servicios institucionales y otros, donde realizan su trabajo los ILSs, necesitamos estudiar más servicios de interpretación para contrastar las estrategias utilizadas entre estos profesionales profundizando en la preparación de estos.

\section{Declaration of conflicting interests}

The author(s) declared no potential conflicts of interest with respect to the research, authorship, and/or publication of this article.

\section{Funding}

The author(s) received no financial support for the research, authorship, and/or publication of this article.

\section{Sobre los autores}

Caridad Rosario Suárez-Martín es doctoranda del Programa de Doctorado de Estudios Lingüísticos y Literarios en sus contextos Socioculturales DELLCOS-ULPGC. Psicopedagoga por la ULPGC en el año 2011. Intérprete de Lengua de Signos Española y Guía Intérprete de personas con sordoceguera desde 2005. 
David Rodríguez-Ruíz es Profesor ayudante doctor siendo su área de conocimiento la Didáctica de la Expresión Corporal Departamento de Educación Física de la ULPGC.

\section{Agradecimientos}

A la Fundación Canaria para las Personas con Sordera y a sus Familias FUNCASOR por la disposición de su Servicio de Interpretación a Lengua de signos Española SILSE-FUNCASOR. En especial a su coordinadora Verónica Rodríguez Cabrera y a la intérprete del servicio Isabel García por sus inestimables colaboraciones.

\section{Referencias}

Alba-Juez, L. (2009). Perspectives on Discourse Analysis. Theory and Practice. Newcastle: Cambridge Scholars Publishing.

Belic, A., Knez, N., Karba, R. y Valencic, V. (2000). Validation of the human muscle model. Proceedings of the 2000 Summer Computer Simulation Conference, 2000, 16-20 July.

Carrasco, L., Sañudo, B., de Hoyo, M.., Pradas, F. y Da Silva, M.E. (2011). Effectiveness of low-frequency vibration recovery method on blood lactate removal, muscle contractile properties and on time to exhaustion during cycling at VO2max power output. European journal of applied physiology, 111(9), 2271-2279.

Dahmane, R., Knez, N., Valencic, V. y Erzen, I. (2000). Tensiomyography, a non-invasive method reflecting the percentage of slow muscle fiber in human skeletal muscles. Book of Abstract: Life Sciencies 2000, 29.

De los Santos, E. y Lara, M.P. (2001). Técnicas de interpretación de lengua de signos. Madrid: Fundación CNSE.

Dean, R. y Pollard, R.Q. Jr. (2001). Application of Demand-Control Theory to Sign Language Interpreting: Implications for Stress and Interpreter Training. The Journal of Deaf Studies and Deaf Education, 6, 1-14.

Dean R. y Pollard R.Q. Jr. (2011). Context-based Ethical Reasoning in Interpreting. The Interpreter and Translator Trainer, 5(1), 155182.

Delisle, A., Larivière, C., Imbeau, D. y Durand, M.J (2005). Physical exposure of sign language interpreters: baseline measures and reliability analysis. European Journal of Applied Physiology, 94, 448-460, 94- 448.

Ditroilo, M., Smith, I.J., Fairweather, M.M. y Hunter, A.M. (2013). Long-term stability of tensiomyography measured under different muscle conditions. Journal of Electromyography and Kinesiology, 23(3), 558-563.

Donner, A., Marshall, M. y Mozrall, J. (2016). Effects of early Exposure to Sign Language on the Biomechanics of Interpreting. Journal of Interpretation., 25,1-14.

Farclough, N. (2012). Critical Discourse Analysis. International Advances in Engineering and Technology (IAET), 7, 461.

Feuerstein, M., Carosella, A., Burrel, L., Marshall, L. y DeCaro, J. (1997). Occupational Upper Extremity Symptoms in Sign Language Interpreters: Prevalence and Correlates of Pain, Function, and Work Disability. Journal of Ocupational Rehabilitation, 7(4), 187205.

Feuerstein, M. y Fitzgerald,T.E. (1992). Biomechanical factors affecting upper extremity cumulative trauma disorders in sign language interpreters. Journal of Occupational and Environmental Medicine, 34(3), 257-264.

Fischer, S., Marshall, M., y Woodcock, K. (2012). Musculoskeletal disorders in sign language interpreters: A systematic review and conceptual model of musculoskeletal disorder development. Work., Vol. 42 (2), 173-184.

Garcia-Garcia, O., Serrano-Gomez, V., Hernandez-Mendo, A. y Tapia-Flores, A. (2016). Assessment of the in-season changes in mechanical and neuromuscular characteristics in professional soccer players. The Journal of sports medicine and physical fitness, $56(6), 14-23$. 
García-Manso, J.M., Rodríguez-Ruiz, D., Rodríguez-Matoso, D., De Saa, Y., Sarmiento, S. y Quiroga, M. (2011). Assessment of muscle fatigue after an ultra-endurancetriathlon using tensiomyography (TMG). Journal of sports sciences, 29(6), 619-625.

García-Manso, J.M. et al. (2012). Effect of high-load and high-volume resistance exercise on the tensiomyographic twitch response of biceps brachii. Journal of Electromyography and Kinesiology, 22(4), 612-619.

Hagberg, M., Stenberg, B. y Sundelin, G. (1987). The use of sign language as an aggravating factor in shoulder tendinitis, a case history of a deaf-mute cleaner. Journal of human ergology, 16(2), 173-178.

Hernández, R., Fernández, C. y Baptista, P. (2010). Metodología de la Investigación. México: McGraw-Hill / Interamericana Editores, S.A. de C.V.

Hunter, A.M. et al.(2012). Assessment of eccentric exercise-induced muscle damage of the elbow flexors by tensiomyography. Journal of Electromyography and Kinesiology, 22(3), 334-341.

Knez, N., Valencic, V. (2000). Proceedings of the ninth Electrocehnical and Computer Science. Conference ERK 21-23, B, 301-304.

Krizaj, D., Simunic, B., y Zagar, T. (2008). Short-term repeatabilityof parameters extracted from radial displacement of muscle belly. Journal of Electromyography and Kinesiology, 18, 645-651.

Lundberg, U. et al. (2002). Effects of experimentally induced mental and physical stress on motor unit recruitment in the trapezius muscle. Work \& Stress, 16(2), 166-178.

Marras, W.S. y Schoenmarklin, R.W. (1993). Wrist motions in industry. Ergonomics, 36(4), 341-351.

Morales López, E (2011). Hacia dónde va el Análisis del Discurso. Tonos Revista Electronica de estudios filologicos, (21), 16-17.

Nicodemus, B., Swabey, L. y Taylor, M.M. (2014). Preparation strategies used by American Sign Language- English interpreters to render President Barack Obama's inaugural address. The Interpreters' Newsletter, 19, 27-44.

Qin, J., Marshall, M., Mozrall, J., y Marschark, M. (2008). Effects of pace and stress on upper extremity kinematic responses in sign language interpreters. Ergonomics, 51 (3), 274-289.

Rey, E., Lago-Peñas, C., Lago-Ballesteros, J., y Casáis, L. (2012). The effect of recovery strategies on contractile properties using tensiomyography and perceived muscle soreness in professional soccer players. The Journal of Strength \& Conditioning Research., 26(11), 3081-3088.

Rodríguez-Matoso, D., Rodríguez-Ruiz, D., Sarmiento, S., Vaamonde, D., Da Silva-Grigoletto, M.E. Y García-Manso, J.M. (2010). Reproducibility of muscle response measurements using tensiomyography in a range of positions. Rev. Andal Med Deporte, 3(3), 81-86.

Rodríguez-Matoso, D., García-Manso, J.M., Sarmiento, S., de Saa, Y., Vaamonde, D., Rodríguez-Ruiz, D. y da Silva-Grigoletto, M.E. (2012). Evaluación de la respuesta muscular como herramienta de control en el campo de la actividad física, la salud y el deporte. Revista Andaluza de Medicina del Deporte, 5(1), 28-40.

Rodríguez Ruiz, D. et al. (2012). The tensiomyography used for evaluating high level beach volleyball players. Revista Brasileira de Medicina do Esporte, 18(2), 95-99.

Shealy, J., Feuerstein, M. y Latko, W (1991). Biomechanical analysis of upper extremity risk in sign language interpreting. Journal of Occupational Rehabilitation., 1(3), 217-225.

Simola, RÁ. et al. (2016). Muscle mechanical properties of strength and endurance athletes and changes after one week of intensive training. Journal of Electromyography and Kinesiology, $\underline{30}, 73-80$.

Simunic, B. y Valencic, V (2001). Procedings of tenth Electrochenical and Computer Science Conference. SlovenianSection IEEE, B, 363-366.

Simunic, B. (2003). Model of longitudinal constractions and transverse deformations in skeletal muscles. Tesis doctoral. Ljubljana: University of Ljubljana Faculty of Electrical Engineering.

Simunic, B. (2012). Between-day reliability of a method for non-invasive estimation of muscle composition. Journal of Electromyography and Kinesiology, 22(4), 527-530.

Stubbs, M. (1983). Análisis del discurso. Análisis sociolingüístico del lenguaje natural. Madrid: Alianza Editorial S.A.. 
Tous-Fajardo, J., Moras, G., Rodríguez-Jiménez, S., Usach, R., Doutres, D.M. y Maffiuletti, NA (2010). Inter-rater reliability of muscle cotractile property measurements using non-invasive tensiomyography. Journal of Electromyography and Kinesiology, 20(4), 761-766.

Valencic, V. y Knez, N. (1997). Measuring of the skeletal muscles dynamic properties. Artific Org, 21, $240-242$.

Valencic, V. (2002). Method for selective and non-invasive detectionof skeletal muscles contraction process. International Application Published under the Patent Cooperation Treaty (PCT), N WO 02/074167 A1.

Winston, E. y Roy, C. (2015). Discourse analysis and sign language. In Schembri, A. y Lucas, C. (Eds.), Sociolinguistics and Deaf Communities (pp. 95-118). Cambridge: Cambridge University Press. 